to the reactions

$$
p \bar{p} \rightarrow n \bar{n}, \quad p n \rightarrow n p,
$$

which are dominated by $\pi$ exchange at small momentum transfers. ${ }^{21,22}$ The results will be published shortly.

${ }^{21}$ J. H. R. Migneron and K. Moriarty, Phys. Rev. Letters 18, 978 (1967)

${ }_{22}$ B. J. Hartley, J. D. Jenkins, R. W. Moore, and K. J. M. Moriarty (to be published).

\section{ACKNOWLEDGMENTS}

We wish to thank Professor P. T. Matthews for encouragement in this work and for a critical reading of the manuscript, I. G. Halliday and L. Saunders for valuable discussions, and R. C. Beckwith for assistance in computing. One of us (B. J. H.) wishes to thank the University of London for a Postgraduate Studentship, and another (R. W. M.) wishes to thank the Science Research Council for a Research Studentship.

\title{
Lagrangian Forms of the Dynamical Theory of Currents*
}

\author{
S. Deser \\ Brandeis University, Waltham, Massachusetts 02154
}

(Received 7 May 1969)

\begin{abstract}
Simple Lagrangian formulations of Sugawara models are given, which directly exhibit the "gauge-component" nature of the currents. It is also shown that there is no corresponding theory for tensor currents.
\end{abstract}

\section{INTRODUCTION}

$\mathrm{T}$ HE dynamical theory of currents ${ }^{1}$ bypasses the usual Lagrangian mechanism of local field theory in favor of a framework involving the physical currents directly. The dynamics governing the currents $j^{\mu}$ is determined by the stress tensor $T^{\mu \nu}$ as a function of $j^{\mu}$ and by the equal-time commutation relations (ETC) among the $j^{\mu}$. Thus, only the Heisenberg equations $\partial_{\mu} j_{\alpha}$ $\equiv i\left[j_{\alpha}, \int T^{0}{ }_{\mu} d^{3} r\right]$ are assigned, with no corresponding Euler-Lagrange equations, which is why the ETC must be separately given. Consistency is no longer checked between Heisenberg and Lagrange equations, but rather between the ETC and the Poincare algebra requirements on $T^{\mu \nu}$. For example, the Schwinger terms in $\left[j_{0}, j_{i}\right]$ ETC give rise to the required $T^{0 i} \partial_{i} \delta(\mathbf{r})$ terms in the $\left[T^{00}, T^{00}\right]$ ETC.

This attractive scheme has been exhaustively analyzed for stress tensors quadratic ${ }^{2}$ in currents carrying $S U_{n} \times S U_{n}$ symmetry; it has been shown to be a particular limit of the corresponding massive YangMills field theories ${ }^{3}$ and also to be equivalent to a Lagrangian theory of spin-zero fields, ${ }^{4,5}$ which furnish representations of the algebra. In Sec. II, we shall give directly a transparent Lagrangian form in terms of the

* Work supported in part by the U. S. Air Force, OAR, under OSR Grant No. 368-67.

${ }^{1}$ H. Sugawara, Phys. Rev. 170, 1659 (1968); C. M. Sommerfield, ibid. 176, 2019 (1968).

${ }^{2}$ For discussion of more general $T^{\mu \nu}$, see S. Deser and J. Rawls, following paper, Phys. Rev. 187, 1935 (1969).

${ }^{3}$ K. Bardakci, Y. Frishman, and M. B. Halpern, Phys. Rev. 170, 1353 (1968).

${ }^{4}$ K. Bardakci and M. B. Halpern, Phys. Rev. 182, 1542 (1968).

${ }^{5} \mathrm{~S}$. Coleman, D. Gross, and R. Jackiw, Phys. Rev. 180, 1355 (1969). currents themselves, which illuminates the basis for the earlier derivations and the close relation between the currents and the gauge or "longitudinal" parts of massive Yang-Mills fields.

Section III deals with possible generalization to higher-spin currents: In view of the close relation of vector currents to gauge components, it might be thought that symmetric tensor currents would correspond to gauge parts of that massive spin-2 field, and, by appropriate extension, of the full gravitational field. However, we show that for a variety of reasons there is no (nontrivial) "dynamical theory of tensor currents."

\section{LAGRANGIAN FORM OF SUGAWARA THEORY}

We exhibit a particularly simple Lagrangian form of the Sugawara model, which preserves the form of $T^{\mu \nu}$. The resulting Euler-Lagrange equations will be the same as the Heisenberg equations in the Sugawara theory, while the current ETC will follow from the canonical commutation relations dictated by the action principle.

Consider first, for simplicity, the Abelian case of a single current $V^{\mu}$, with

$$
T^{\mu \nu}=V^{\mu} V^{\nu}-\frac{1}{2} \eta^{\mu \nu} V_{\alpha} V^{\alpha},
$$

where, as throughout, all required symmetrization is understood, and the usual over-all constant $C$ is set to unity. This stress tensor is the variation of an "action"

$$
I_{1}=-\frac{1}{2} \int d^{4} x(-g)^{1 / 2} g^{\mu \nu} V_{\mu} V_{\nu}
$$


with respect to $g_{\mu \nu},{ }^{6}$ according to the definition

$$
T^{\mu \nu}=2 \delta I / \delta g_{\mu \nu} .
$$

We now add to $I_{1}$ a metric-independent kinetic part $I_{0}$, which therefore does not contribute to $T^{\mu \nu}$ :

$$
I_{0}=-\frac{1}{2} \int d^{4} x F^{\mu \nu}\left(\partial_{\mu} V_{\nu}-\partial_{\nu} V_{\mu}\right)
$$

Here $F^{\mu \nu}$ is a contravariant antisymmetric tensor density, and the ordinary curl of $V_{\mu}$ is of course a tensor under general coordinate transformations. In varying the total action $I=I_{0}+I_{1}, F^{\mu \nu}$ and $V_{\mu}$ are independent, as in the usual first-order formalism; i.e., the relation between $F^{\mu \nu}$ and $V_{\mu}$ is given $a$ posteriori by the field equations, which read

$$
0=\partial_{\mu} V_{\nu}-\partial_{\nu} V_{\mu}, \quad \partial_{\nu} F^{\mu \nu}+V^{\mu}=0 .
$$

These equations differ in one crucial way from those of massive spin-1 theory, namely, $F^{\mu \nu}$ is missing in the curl equation; this is of course due to omission in our action $I$ of the usual $\frac{1}{4} \int F_{\mu \nu} F^{\mu \nu}$ term for this field. We shall trace the purely longitudinal character of the currents to the absence of this term; conversely, dropping the $V^{2}$ mass term and keeping $F^{2}$ would lead to the - purely transverse-Maxwell theory. Conservation $^{7}$ of $V^{\mu}$ follows from the identity $\partial_{\mu \nu}{ }^{2} F^{\mu \nu} \equiv 0$. Thus both the equations of motion for $V^{\mu}$ (that it is divergence- and curl-free) and the stress tensor are the same as in Sugawara theory. However, we now have a normal canonical theory and may also obtain the conjugate pairs of variables, which will then yield the current ETC. To accomplish this, we must solve the constraint equations present in (2.4a) and remove the redundant variables from the action. In terms of $E^{i} \equiv F^{0 i}$, we have

$$
0=\nabla \times \mathbf{V}, \quad V_{0}=\nabla \cdot \mathbf{E} .
$$

The general solution of the differential constraint is of course $\mathbf{V}=\nabla S$, with $S$ an arbitrary scalar field. The total action then reduces to the form

$$
I=\int d^{4} x\left\{\nabla \cdot \mathbf{E} \partial_{0} S-\frac{1}{2}\left[(\nabla S)^{2}+(\boldsymbol{\nabla} \cdot \mathbf{E})^{2}\right]\right\}
$$

\footnotetext{
${ }^{6}$ In general, the form of $T^{\mu \nu}$ of course depends on the choice of tensor (density) behavior of the matter variables: Since the ratio of $V^{\mu} V^{\nu}$ to $\eta^{\mu \nu} V^{2}$ in (2.1) must be as given (Ref. 1), there are only two possible choices-the one in the text ( $V_{\mu}$ a covariant vector) or $V^{\mu}$ a contravariant vector density. The latter leads to the alternative Lagrangian form of the Appendix. There are also basic geometrical reasons for these choices.

${ }^{7}$ Formally, $F^{\mu \nu}$ is a Lagrange multiplier that yields the cur condition. One may ask whether the Lorentz gauge condition $\partial_{\mu} V^{\mu}=0$ may be replaced by other covariant ones such as the Dirac gauge $\left(V^{2}=1\right)$. This appears possible, by appropriate use of other multipliers, but the quadratic $T^{\mu \nu}$ form is then lost, being particular to the linear nature of Lorentz gauge. Noncovariant gauges, such as $\boldsymbol{\nabla} \cdot \mathbf{V}=0$, of course yield trivial solutions.
}

and we have the single degree of freedom $(\boldsymbol{\nabla} \cdot \mathbf{E}, S)$, with

$[\boldsymbol{\nabla} \cdot \mathbf{E}(\mathbf{r}), S(0)]=-i \delta(\mathbf{r}),[\boldsymbol{\nabla} \cdot \mathbf{E}, \boldsymbol{\nabla} \cdot \mathbf{E}]=0=[S, S]$,

which may be written as the Abelian current algebra $\left[V^{0}(\mathbf{r}), V^{i}(0)\right]=-i \partial_{i} \delta(\mathbf{r}),\left[V^{0}, V^{0}\right]=0=\left[V^{i}, V^{j}\right]$.

Note that the "magnetic field" $F^{i j}$ and the transverse part of $\mathbf{E}$ have both disappeared completely from the action. Thus, only the longitudinal degree of freedom remains (as evidenced also by the longitudinal nature of $\left.\left[V^{0}, V^{i}\right]\right)$, without need for a limiting procedure from the full massive theory. Likewise, the scalar field representation is not needed in presenting the original Lagrangian form $I=I_{0}+I_{1}$, but first arises upon solution of the constraints.

Analysis of the non-Abelian case follows the same lines; the difference is that $\partial_{\mu}$ is now replaced by the covariant combination

$$
\partial_{\mu} \rightarrow D_{\mu}{ }^{a c} \equiv\left(\delta^{a c} \partial_{\mu}-\frac{1}{2} c_{a b c} J_{\mu}^{b}\right),
$$

where $c_{a b c}$ are the structure constants, and we keep $C=1$. The corresponding action, suppressing internal indices, is

$$
I=-\frac{1}{2} \int d^{4} x\left[F^{\mu \nu} \cdot\left(D_{\mu} J_{\nu}-D_{\nu} J_{\mu}\right)+J_{\mu} \cdot J^{\mu}\right],
$$

with field equations $\left(\bar{D}_{\mu}^{a c} \equiv \delta^{a c} \partial_{\mu}-C_{a b c} J_{\mu}^{b}\right)$,

$$
0=D_{\mu} J_{\nu}-D_{\nu} J_{\mu}, \quad \bar{D}_{\nu} F^{\mu \nu}+J^{\mu}=0,
$$

while the stress tensor is again (2.1), with internal index summation understood. Conservation of $J^{\mu}$ follows from the fact that $\left[\bar{D}_{\mu}, \bar{D}_{\nu}\right] F^{\mu \nu}=0$ and $\bar{D}_{\mu} J^{\mu}=\partial_{\mu} J^{\mu}$. The constraints now read

$$
0=D_{i} J_{j}-D_{j} J_{i}, \quad J_{0}=\overline{\mathbf{D}} \cdot \mathbf{E} .
$$

To illustrate the solution of the curl equation, we consider for definiteness the algebra of $S U_{2}$. While the current $J_{i}$ is no longer longitudinal, it still depends only on a scalar (isovector) $S^{a}$, since there are two constraints on its three (spatial) components. Its most general form (linear in $\partial_{i}$ ) is

$$
J_{i}=a S_{, i}+b_{, i} S+c S \times S_{, i},
$$

where $a, b, c$ are scalar functions of $S^{2} \equiv S^{a} S^{a}$; vector notation is used in isospace. Inserting this form into the (isovector) condition $D_{i j} \equiv \partial_{i} J_{j}-\partial_{j} J_{i}-J_{i} \times J_{j}=0$, we obtain, after a simple calculation, the conditions on the coefficients $a, b, c$. These may be conveniently read off from the requirements $S \cdot D_{i j}=0=S \times D_{i j}$ (which are of course equivalent to $\left.D_{i j}=0\right)$;

$$
\begin{aligned}
a^{2}+c^{2} S^{2}-2 c & =0, \\
2\left(a^{\prime}-b^{\prime}\right)+a c+2 c S^{2} b^{\prime} & =0, \\
2\left(c+S^{2} c^{\prime}\right)-a^{2}-2 a S^{2} b^{\prime} & =0,
\end{aligned}
$$


where the primes denote differentiation with respect to the argument $S^{2}$. The existence of a family of solutions corresponds to the freedom of canonical transformations of the form $S=\hat{S} f\left(\hat{S}^{2}\right)$, which in turn correspond to different conjugate momenta. The result of Ref. 4 is included:

$$
\frac{1}{2} J_{i}=S \times S_{, 2}+S^{0}{ }_{i} S-S^{0} S_{, i}, \quad S^{0} \equiv\left(1-S^{2}\right)^{1 / 2} .
$$

Some other simple forms are

$$
\frac{1}{2} J_{i}=\left(S_{, i}+S \times S_{, i}\right)\left(1+S^{2}\right)^{-1},
$$

in which the coefficient of $S$ vanishes. The choice

$$
\frac{1}{2} J_{i}=S_{, i}+(\ln f)_{, i} S+f S \times S_{, i},
$$

where $S^{2} f^{2}-f+1=0$, has the leading Abelian form $J \sim S_{, i}$. Finally, a particularly simple form, which is singular as it stands in the Abelian limit, is

$$
\frac{1}{2} J_{i}=S^{-2} S \times S_{, i} .
$$

On the other hand, it is not possible to eliminate the characteristic "rotation" term $S \times S_{, i}$, since $c=0$ implies also $a=b=0$.

The extension to $S U_{2} \times S U_{2}$ is immediate, if we use the remark ${ }^{8}$ that this theory is simply the direct sum of two $S U_{2}$ theories in terms of $J^{\mu}{ }_{ \pm} \equiv \frac{1}{2}\left(V^{\mu} \pm A^{\mu}\right)$, so that $V^{\mu}$ and $A^{\mu}$ are represented by sums and differences of solutions (2.14) of the form (2.14) in terms of two independent scalars $S_{+}$and $S_{-}$.

Having obtained $J_{i}$ in terms of the single degree of freedom $S$, we can find the momentum $I$ conjugate to $S$ as the coefficient of $\partial_{0} S$ in the kinetic energy; this agrees with that given in Ref. (4), for the particular representation (2.14a). The $S U_{2}$ algebra of the $J_{\mu}$ then follows from the canonical $[\Pi, S]$ ETC, with the constraint $J_{0}=\overline{\mathbf{D}} \cdot \mathbf{E}$. Thus, the Sugawara theory is completely equivalent to ordinary scalar field models with appropriate self-interaction corresponding to the solutions of (2.13). An alternative Lagrangian formulation, with the scalar representations built in directly, is given in the Appendix.

An additional advantage of the Lagrangian form is that the Lorentz covariance condition

$$
i\left[T^{00}(\mathbf{r}), T^{00}(0)\right]=\left[T^{0 i}(\mathbf{r})+T^{0 i}(0)\right] \partial_{i} \delta^{3}(\mathbf{r}),
$$

along with the other relations $i\left[T^{0 \mu}(\mathbf{r}), T^{\lambda \sigma}(0)\right]$ connected with the Poincare algebra, is immediately obtained merely by examining the metric dependence of the action. ${ }^{9,10}$ In this connection, we mention two problems occurring in stress tensor ETC for local fields which are also present in Sugawara models. The first is that Lorentz covariance requires ${ }^{9}$ the presence of Schwinger terms of the form $\partial^{3}{ }_{i j k} \delta(\mathbf{r})$ in the vacuum expectation of the $\left[T^{00}, T^{0 i}\right]$ and $\left[T^{0 i}, T^{j k}\right]$ ETC. On the

\footnotetext{
${ }^{8}$ R. Dashen and Y. Frishman, Phys. Rev. Letters 22, 572 (1969).

${ }^{9}$ D. G. Boulware and S. Deser, J. Math. Phys. 8, 1468 (1967).

${ }^{10} \mathrm{~S}$. Deser and I. K. Morrison, J. Math. Phys. (to be published).
}

other hand, it is clear that no such terms can arise from the algebra of currents, since these contain only ${ }^{11} \partial_{i} \delta(\mathbf{r})$. This problem of Sugawara theory has been noted also in Ref. 5, but one suggested remedy, that the currents be "spread" in a nonspacelike fashion,

$$
j^{2} \rightarrow j(x+\epsilon) j(x-\epsilon), \quad \epsilon^{0} \neq 0,
$$

seems too drastic, since time locality is lost and the dynamical scheme is no longer well defined. It would seem that, just as for free fields, the use of purely spacelike spreading prescriptions should suffice. The second problem is that, while Lorentz invariance requires that $\left\langle T^{\mu \nu}\right\rangle=\lambda \eta^{\mu \nu}$ (covariance of vacuum selfstress), direct computation yields two different (infinite) constants $^{9}\left\langle T^{i j}\right\rangle=\delta^{i j} \lambda,\left\langle T^{00}\right\rangle=\lambda^{\prime}$ for any free field, for example. Consequently, in the vacuum expectation of the Sugawara-model ETC,

$$
i\left[T^{0 i}(\mathbf{r}), T^{00}(0)\right]=\left[T^{i j}(\mathbf{r}) \partial_{j}+T^{00}(0) \partial_{i}\right] \delta(\mathbf{r}),
$$

there should be no $\partial_{i} \delta(\mathbf{r})$ terms, whereas they are present by direct calculation. ${ }^{12}$

\section{TENSOR CURRENTS}

Just as the vector-current models represent the gauge (or longitudinal, lower-spin) parts of vector gauge theories, one might expect that models in which $T^{\mu \nu}$ is constructed from tensor currents would describe the gauge parts of the corresponding tensor theory. In particular, representations of the linearized or full gravitational gauge group might be expected to arise from appropriate choices of $T^{\mu \nu}$ built from symmetric tensor currents $h_{\alpha \beta}$. We shall see, however, that no nontrivial Sugawara models exist for tensor (and probably also higher-spin) currents.

The intermediate case in which the "currents" are antisymmetric tensors $F_{\mu \nu}$, with the usual Maxwell $T_{\mu \nu}(F)$, is just ${ }^{1}$ Maxwell (or Yang-Mills) theory. ${ }^{13}$ One could of course reach the "canonical" form of this model by appending to the "action"

$$
I_{1}=\frac{1}{4} \int F^{\mu \nu} F^{\alpha \beta} g_{\mu \alpha} g_{\nu \beta}(-g)^{-1 / 2} d^{4} x
$$

the kinetic term $-\frac{1}{2} \int F^{\mu \nu}\left(\partial_{\mu} A_{\nu}-\partial_{\nu} A_{\mu}\right)$, which does not alter $T^{\mu \nu}$. We note that the quantum Maxwell field (but not non-Abelian ones) can be defined, in this framework,

\footnotetext{
${ }^{11}$ Only if $T^{\mu \nu}$ depends on a canonical tensor field $h_{\mu \nu}$, which does have $\partial^{3} \delta(\mathbf{r})$ ETC, can one hope to reproduce the Schwinger terms. However, we shall see in Sec. III that $T^{\mu \nu}$ bilinears in such $h_{\mu \nu}$ are not possible, while a $T^{\mu \nu}$ linear in $h_{\mu \nu}$ of course loses the Poincaré part of the ETC.

12 D. Corrigan and J. G. Kuriyan [Phys. Rev. 183, 1472 (1969)] have also considered this problem, from another point of view. ${ }_{13}$ While the Sugawara form thus avoids explicit introduction of the vector potential, the latter would enter with minimal coupling to charged currents through the ETC of the matter variables, although $T^{\mu \nu}$ would still be $A_{\mu}$-independent.
} 
by the sole algebraic requirements that ${ }^{14} T_{\alpha}^{\alpha}=0$, $T_{\mu \alpha} T_{\nu}^{\alpha}=\frac{1}{4} \eta_{\mu \nu} T_{\alpha \beta} T^{\alpha \beta}$, since these are known to imply that $T_{\mu \nu}$ has the Maxwell form in terms of a tensor $F_{\mu \nu}=-F_{\nu \mu}$. The field equations then follow ${ }^{1}$ entirely from the Lorent $z$ covariance requirement that $\left[F_{0 i}, F_{k l}\right]$ have at least a $c$-number Schwinger term. Classically, such a term is not manifestly needed, and a further assumption is necessary (beyond conservation of $T^{\mu \nu}$ ) to obtain Maxwell's equations.

Returning to spin 2, the gauge part of the field (in the linear case) is known to consist of tensors of the form

$$
h_{\mu \nu}=\zeta_{\mu, \nu}+\zeta_{\nu, \mu} \text {, }
$$

where $\zeta_{\mu}$ is an arbitrary vector. ${ }^{15}$ Further, if there were a limit from the massive field, for which $h_{\mu \nu, \nu}=0=h_{\alpha \alpha}$, one would expect the $\zeta_{\mu}$ to obey the gauge conditions $\square \zeta_{\mu}=0=\zeta_{\mu, \mu}$. The gauge field is then precisely the Maxwell field in Lorentz gauge. However, the limit from massive theory (corresponding to $F_{\mu \nu} \rightarrow 0$ in the spin-1 case $)$ is $\Gamma_{\alpha \beta}{ }^{\mu}=\frac{1}{2}\left(h_{\mu \alpha, \beta}+h_{\mu \beta, \alpha}-h_{\alpha \beta, \mu}\right) \rightarrow 0$, which implies that $h_{\mu \nu}$ itself vanishes. (The gauge-invariant field strength is actually the "curvature" $\Gamma_{\alpha \beta, \nu}^{\mu}-\Gamma_{\alpha \nu, \beta}^{\mu}$.) Thus, the fact that one obtains both "vanishing curvature" [Eq. (3.1)] and "Cartesian coordinates," $\zeta_{\mu, \nu}+\zeta_{\mu, \nu}=0$, is due to the non-gauge invariance of $\Gamma_{\alpha \beta}^{\mu}$. The above argument can be borne out by several explicit calculations as well. Thus, starting with the appropriate (traceless ${ }^{14}$ ) $T^{\mu \nu}$, it may be put in the form

$$
T_{\mu \nu}=h_{\mu \alpha} h^{\alpha}{ }_{\nu}-\frac{1}{4} \eta_{\mu \nu} h_{\alpha \rho} h^{\alpha \beta}
$$

by appropriate choice of the trace of $h_{\mu \nu}$. A full calcula$\operatorname{tion}^{16}$ of the Heisenberg equations for $h_{\mu \nu, \alpha}$, using, for example, the minimal requirements from Lorentz invariance on the ETC, namely,

$$
\begin{aligned}
i\left[h_{00}(\mathbf{r}), h_{0 i}(0)\right]=\lambda \nabla^{2} \partial_{i} \delta(\mathbf{r}), & \\
& i\left[h_{0 i}(\mathbf{r}) h_{l m}(0)\right]=\lambda \partial^{3}{ }_{i l m} \delta(\mathbf{r}),
\end{aligned}
$$

together with $\partial_{i} \delta(\mathbf{r})$ terms, shows that only the trivial $h_{\mu \nu}=0$ possibility is permitted. It is also not possible to get a Lagrangian form here: No kinetic term can be introduced that does not contribute to $T_{\mu \nu}$, because of the tensor character of $h_{\mu \nu}$ (any term of the form $\Gamma^{\mu}{ }_{\alpha \beta} h_{\alpha \beta, \mu}$ will contribute to $\left.T^{\mu \nu}\right)$. The difficulty may be seen in a purely dimensional way as well, in attempting to limit from the massive theory: The ETC among the $h_{\alpha \beta}$ of massive spin-2 theory ${ }^{17}$ contain terms proportional to $m^{-4} \partial^{3} \delta(\mathbf{r})$. Thus the $m \rightarrow 0$ limit $^{3}$ must be such as to absorb an $m^{4}$ factor in the field variables (say, by

\footnotetext{
${ }^{14}$ We mention incidentally that tracelessness of $T^{\mu \nu}$ for massless fields of spin $>0$ is connected with their pure helicity character, so that no spin- 0 states are generated from the vacuum by $T^{\mu \nu}$. For zero spin, this property is absent, and correspondingly $T_{\alpha} \alpha \neq 0$. The fact that the Yang-Mills field's $T_{\alpha}{ }^{\alpha}$ vanishes is thus a strong indication that it really describes massless particles.

${ }^{15}$ An appropriate choice of $h_{\mu \nu}$ absorbs terms $\sim \eta_{\mu \nu} \zeta_{\alpha, \alpha}$.

${ }_{16}$ This has been carried out for general assumptions on linear ETC among $h_{\mu \nu}$ components consistent with the Lorentz-invariance requirements by $\mathrm{P}$. Mansbach (unpublished).

${ }^{17}$ See, e.g., S. Deser, J. and S. Trubatch, Can J. Phys. 44, 1715

(1966); or S. J. Chang, Phys. Rev. 148, 1259 (1966).
}

redefining $\tilde{h}=m^{2} h$ ). However, the mass part of $T^{\mu \nu}$ goes as $m^{2} h h$ and thus would diverge in terms of $\tilde{h}$.

Finally, the same difficulties would hold in taking the limit of "massive" general relativity, ${ }^{18}$ as the $\Gamma^{\mu}{ }_{\alpha \beta} \rightarrow 0$ limit would still lead to the trivial solution $h_{\mu \nu}=0$. The absence of a tensor Sugawara theory is certainly not to be considered a physical drawback, however, since the only known physical tensor current is the stress tensor itself. ${ }^{19}$

\section{APPENDIX}

We give here an alternative Lagrangian formulation of the Sugawara model, which is complementary to the one of Sec. II. The latter emphasizes the role of the current as the longitudinal part of a gauge field; the present one will bring out directly the scalar representations of the model. In the Abelian case, we consider the action

$$
I=-\int\left[j^{\mu} \partial_{\mu} \phi-\frac{1}{2} j^{\mu} j^{v} g_{\mu \nu}(-g)^{-1 / 2}\right] d^{4} \mathrm{x}
$$

where $j^{\mu}$ is now defined as a contravariant vector density and $\phi$ is a scalar. The kinetic term is metricindependent, and we get the Sugawara stress tensor

$$
T^{\mu \nu}=j^{\mu} j^{\nu}-\frac{1}{2} \eta^{\mu \nu} j^{2},
$$

along with the equations of motion, from varying $j_{n}$ and $\phi$ independently:

$$
j_{\mu}=\partial_{\mu} \phi, \quad \partial_{\mu} j^{\mu}=0
$$

which is precisely the Abelian Sugawara theory. The canonical commutators

$$
\left[j^{0}, \phi\right]=i \delta(\mathbf{r}), \quad\left[j^{0}, j^{0}\right]=0=\left[j^{i}, j^{k}\right]
$$

then clearly yield the Abelian current algebra.

In the non-Abelian case of, say, $S U_{2}$ the action must be modified to yield the field equations $D_{\mu} J_{\nu}-D_{\nu} J_{\mu}$ $=0=\partial_{\mu} J^{\mu}$. The curl equation may be simply incorporated into the theory by writing the action as

$$
I=-\int d^{4} x\left[J^{\mu}\left(a S_{, \mu}+b_{, \mu} S+c S \times S_{, \mu}\right)-\frac{1}{2} J^{2}\right],
$$

where $a, b$, and $c$ satisfy the conditions (2.13). This clearly builds the curl condition into the field equations

$$
J_{\mu}=a S_{, \mu}+b_{, \mu} S+c S \times S_{, \mu}
$$

by construction. The conservation of $J_{\mu}$ then follows from varying $S$. Likewise, the canonical variables may be read off from the kinetic term, once the constraints $(\mu=i)$ in Eq. (A6) are employed. The action (A5) is just the first-order form equivalent of the second-order Lagrangian of Ref. 4.

${ }^{18}$ V. I. Ogievetsky and I. V. Polubarinov, Ann. Phys. (N. Y.) 35, 167 (1965)

${ }_{19}$ There is a conserved fourth-rank tensor in the spin-2 theory [L. Bel, Compt. Rend. 248, 1297 (1959)], which might perhaps yield an appropriate limit, but its physical significance is not very clear. 